\title{
Resultado del tratamiento de la hemorragia subaracnoidea debida a rotura de aneurismas cerebrales
}

\author{
Ma.I. González-Pérez
}

Servicio de Medicina Intensiva. Complejo Hospitalario de León.

Resumen

Objetivo. Evaluar a los pacientes con hemorragia subaracnoidea (HSA) aneurismática confirmada, en el Hospital de León, durante los años 2001 a 2003.

Material y métodos. El protocolo de tratamiento incluye: traslado rápido al Centro de Referencia, control de la tensión arterial e infusión de nimodipino, arteriografía y tratamiento endovascular en las primeras 72 horas del ingreso. Si no es posible embolizar, cirugía lo más precoz posible. Manejo agresivo antiisquemia y utilización de doppler transcraneal para el diagnóstico y seguimiento del vasoespasmo.

Los 54 pacientes ingresados vivos con el diagnóstico principal de HSA se clasificaron según las escalas de Hunt-Hess y Fisher, al ingreso. El seguimiento se hizo hasta el alta del Hospital; analizando morbilidad (presencia de un déficit neurológico al alta, de cualquier grado, diferente a la parálisis de un par craneal) y la mortalidad. Se consideró estadísticamente significativa una $\mathbf{p}<\mathbf{0 . 0 5}$.

Resultados. Durante el periodo de estudio, el $86 \%$ de los pacientes que ingresaron con HSA lo hicieron en las 24 horas siguientes al sangrado. Cinco pacientes no fueron tratados por presentar HSA masiva con HuntHess V, de los que fallecieron 4. En los 49 (90,74\%) restantes la embolización se llevó a cabo con éxito en 28 (54\%) y en $21(38 \%)$ no se pudo realizar por las características del aneurisma; se operaron en las siguientes 72 horas.

Seis pacientes $(16,8 \%)$ embolizados y $3(14,2 \%)$ operados tuvieron secuelas neurológicas $(p>0,05)$. Fallecieron $3(14,2 \%)$ enfermos con tratamiento endovascular y $4(\mathbf{1 9 \%})$ quirúrgicos $(\mathrm{p}>0,05)$.

La frecuencia de vasoespasmo fue de un $20 \%$ y la de resangrado de un $16 \%$.

La estancia en UCI de los pacientes embolizados fue de 6,1 días y la de los pacientes con tratamiento quirúrgico fue de 8,8 . $(p<0.05)$.

Recibido;18-10-05. Aceptado: 20-11-05
Conclusión. El tratamiento endovascular se puede realizar con éxito en más de la mitad de los pacientes. Hay relación entre el nivel de conciencia al ingreso y el pronóstico. No encontramos diferencias significativas, desde el punto de vista estadístico, en la morbi-mortalidad entre los pacientes embolizados y los intervenidos quirúrgicamente, pero sí en cuanto a la estancia en UCI (menor en los pacientes tratados por procedimiento endovascular).

PALABRAS CLAVE: Hemorragia subaracnoidea. Aneurisma. Embolización. Resultado.

Results of treatment of subarachnoid haemorrhage due to a ruptured cerebral aneurysm

Summary

Objective. This study was designed to evaluate patients with confirmed aneurismal SAH (subarachnoid haemorrhage) in León Hospital between 2001 and 2003.

Materials and methods. The treatment protocol includes: rapid transfer to the reference centre, blood pressure control and nimodipine infusion, arteriography and endovascular treatment within the first 72 hours after admission. Surgery is performed as soon as possible if embolization is not possible. Aggressive antiaeschemic handling and transcranial Doppler are used for diagnosis and vasospasm monitoring.

The patients were classified on admission according to the Hunt-Hess scale. Monitoring continued until discharge, and morbidity (any degree of neurological deficiency on discharge, not paralysis of a cranial pair) and mortality were analysed. $P<0.05$ was considered statistically significant.

Results. During the study period 54 patients had

Abreviaturas. HSA: Hemorragia subaracnoidea. ICU: Intensive Care Unit. TAM: Tensión arterial media. UCI: Unidad de Cuidados Intensivos. 
SAH on admission, $86 \%$ of whom were admitted to Intensive Care Unit 24 hours after bleeding. Five patients were not treated as they had massive SAH with Hunt-Hess V. Four of them died . Of the remaining 49 patients $(90.74 \%)$ embolization was successful in 28 $(54 \%)$ but $21(38 \%)$ were not embolized due to the characteristics of the aneurysm; they were operated on within the following 72 hours.

Six embolized $16.8 \%)$ and $3(14.2 \%)$ patients operated on had neurological sequelae $(p>0.05)$. Three endovascularly treated patients $(14.2 \%)$ and $4(19 \%)$ surgically treated died $(p>0.05)$. The frequency of clinical vasospasm was $20 \%$ an rebleeding occurred in $16 \%$. The length of stay in the ICU of embolized patients was significantly shorter than the operated patients $(6.1 \mathrm{vs}$ 8.8 )days, $p<0.05$.

Conclusion. The endovascular treatment can be successfully carried out in over half of the patients with ruptured aneurysms. There is a significant relationship between the degree of consciousness on admission and the prognosis. We found no statistically significant differences in morbid-mortality between the embolized patients and those undergoing conventional surgery. However, there was a difference in length of stay in the ICU (shorter in patients treated by endovascular coiling).

KEY WORDS: Subarachnoid haemorrhage. Aneurysm. Embolization. Result.

\section{Introducción}

La hemorragia subaracnoidea (HSA) debida a aneurismas rotos es una urgencia neurológica con alta morbi-mortalidad que mantiene una incidencia estable (6-8 casos / 100.000 habitantes / año $)^{20}$.

Los supervivientes tienen la posibilidad de retornar a su vida normal, lo cual obliga a los médicos a reevaluar constantemente el tratamiento.

Los protocolos de tratamiento varían considerablemente entre unos centros y otros. En los últimos años hay una tendencia a mantener una estrategia uniforme, especialmente en lo que se refiere al traslado rápido al Centro de Referencia para intentar prevenir el resangrado. La atención en la Unidad de Cuidados Intensivos y el tratamiento precoz quirúrgico o endovascular del aneurisma roto es lo principal durante las horas que siguen a la clínica inicial ${ }^{25}$.

Este manejo abarca también un tratamiento agresivo anti-isquemia con nimodipino más hemodilución para minimizar el deterioro debido a la isquemia

\section{Objetivos}

Evaluar los resultados del manejo de los pacientes con
HSA aneurismática en el Hospital de León, tras el inicio de la terapia endovascular precoz.

\section{Material y métodos}

El Hospital de León es el Centro de Referencia de Neurocirugía de una población de alrededor de 500.000 habitantes.

Pacientes. Se hizo una evaluación retrospectiva de todos los pacientes admitidos en la Unidad de Cuidados Intensivos del Hospital de León, con el diagnóstico principal de hemorragia subaracnoidea aneurismática durante los años 2001 al 2003.

Se excluyeron los pacientes con HSA traumática o no aneurismática.

Información básica. Se recogió la siguiente información de cada paciente: edad, sexo, APACHE II, gravedad del déficit neurológico inicial, clasificado según la escala de Hunt-Hess ${ }^{8}$ descrita por el Médico Intensivista que lo exploró al ingreso. La cantidad de sangre en el TC inicial se clasificó según el grado de Fisher ${ }^{3}$, basándose en el informe radiológico.

En pacientes transferidos de otro Centro el grado se basó en la interpretación por el médico especialista en Medicina Intensiva.

Protocolo de manejo para pacientes con HSA. El protocolo de tratamiento incluye:

1) traslado rápido al Centro de Referencia. 2) analgesia. 3) mantenimiento de TAM (tensión arterial media) en torno a 90-100. 4) infusión de nimodipino. 5) arteriografía y tratamiento endovascular en las primeras 72 horas del sangrado. 6) si no es posible embolización, tratamiento quirúrgico, lo más precoz posible. 7) manejo agresivo antiisquemia, usando nimodipino y hemodilución, hipervolémica, hipertensiva, una vez excluido el aneurisma. 8) uso de doppler transcraneal para el diagnóstico y seguimiento del vasoespasmo (llevado a cabo diariamente en pacientes con velocidades altas o cambios en la exploración neurológica)

Registramos lo siguiente para cada paciente: tiempo de intervención quirúrgica / embolización del aneurisma y el total de días pasados en la Unidad de Cuidados Intensivos.

Determinación del resultado. El resultado se evaluó al alta de la hospitalización aguda. Consideramos como morbilidad cualquier déficit neurológico diferente de la parálisis de un par craneal (basándonos en la exploración del Neurocirujano al alta).

Análisis estadístico. El resultado fue dicotomizado en bueno o malo.

Los factores pronósticos evaluados fueron grado de Fisher (figura 2) y Hunt-Hess (figura 1) en el momento de la admisión.

Se comparó el tratamiento quirúrgico precoz con el 


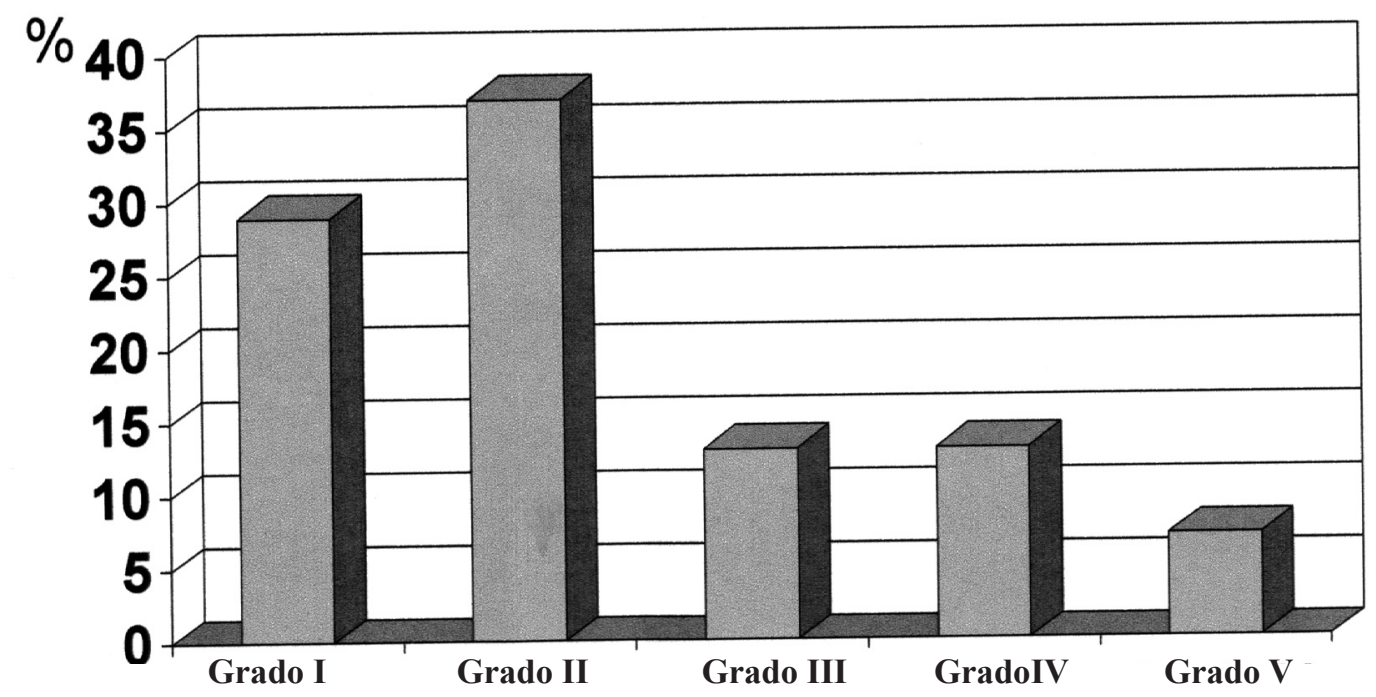

Figura 1. Clasificación del grado de afectación neurológica según la escala de Hunt-Hess.

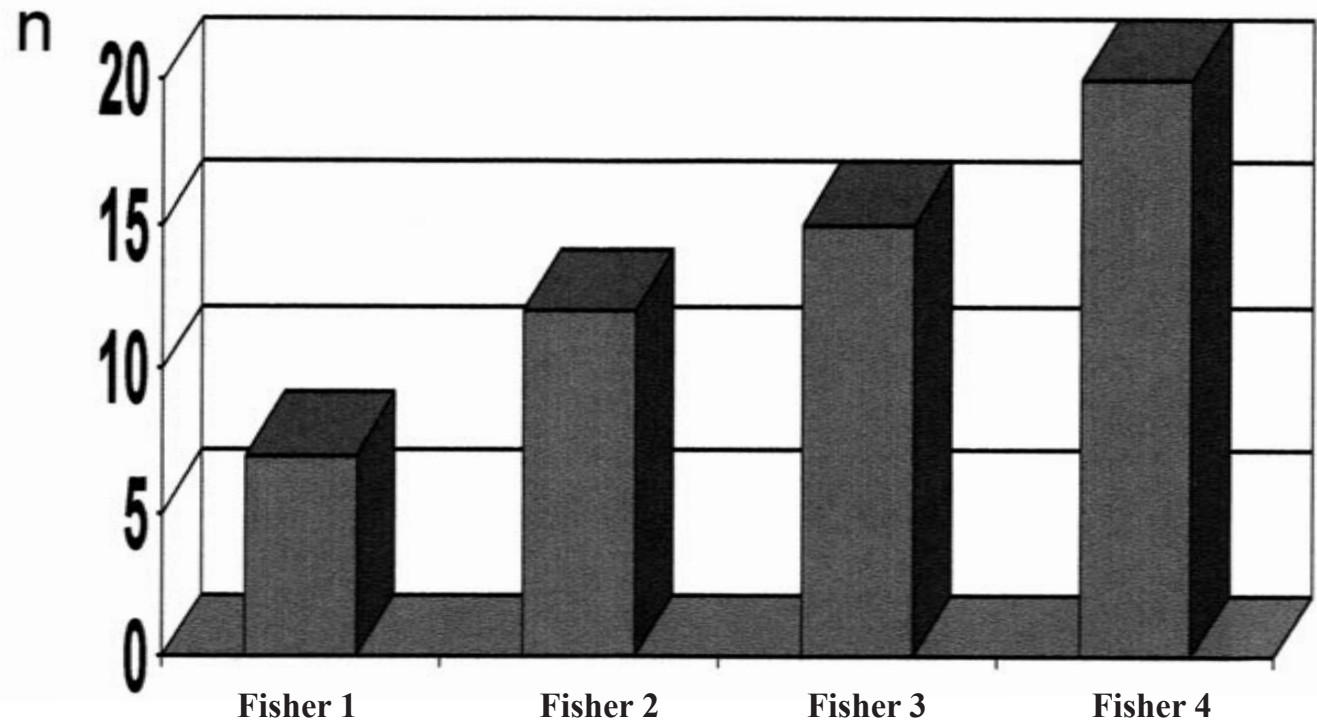

Figura 2. Clasificación del sangrado intracraneal según la escala de Fisher.

endovascular en términos de pronóstico y estancia en UCI.

Se utilizó $\chi 2$ para comparar frecuencias y test de Wilcoxon para comparar las características demográficas de los pacientes, cuando fue apropiado.

Todos los valores fueron expresados en media. Se consideró una $\mathrm{p}<0.05$ como significativa.

\section{Resultados}

Pronóstico. Hubo 54 pacientes admitidos vivos en la UCI con el diagnóstico de HSA aneurismática desde enero de 2001 hasta diciembre de 2003 (ambos incluidos).
La edad media fue de 52,87. 29 (54\%) eran mujeres. 29 pacientes (54\%) tuvieron una buena recuperación neurológica, 14 pacientes (26\%) sufrieron algún tipo de morbilidad y $11(20 \%)$ murieron.

La mortalidad global fue $20 \%(n=11)$. Al alta presentaban morbilidad 26\% $(n=14)$. Los pacientes con pobres resultados presentaban grados altos de Hunt-Hess. (figura $3)$.

La tabla 1 muestra la relación entre la localización del aneurisma roto y el resultado (no hubo asociación entre localización y pronóstico).

De los 25 pacientes con evolución desfavorable: en 5 


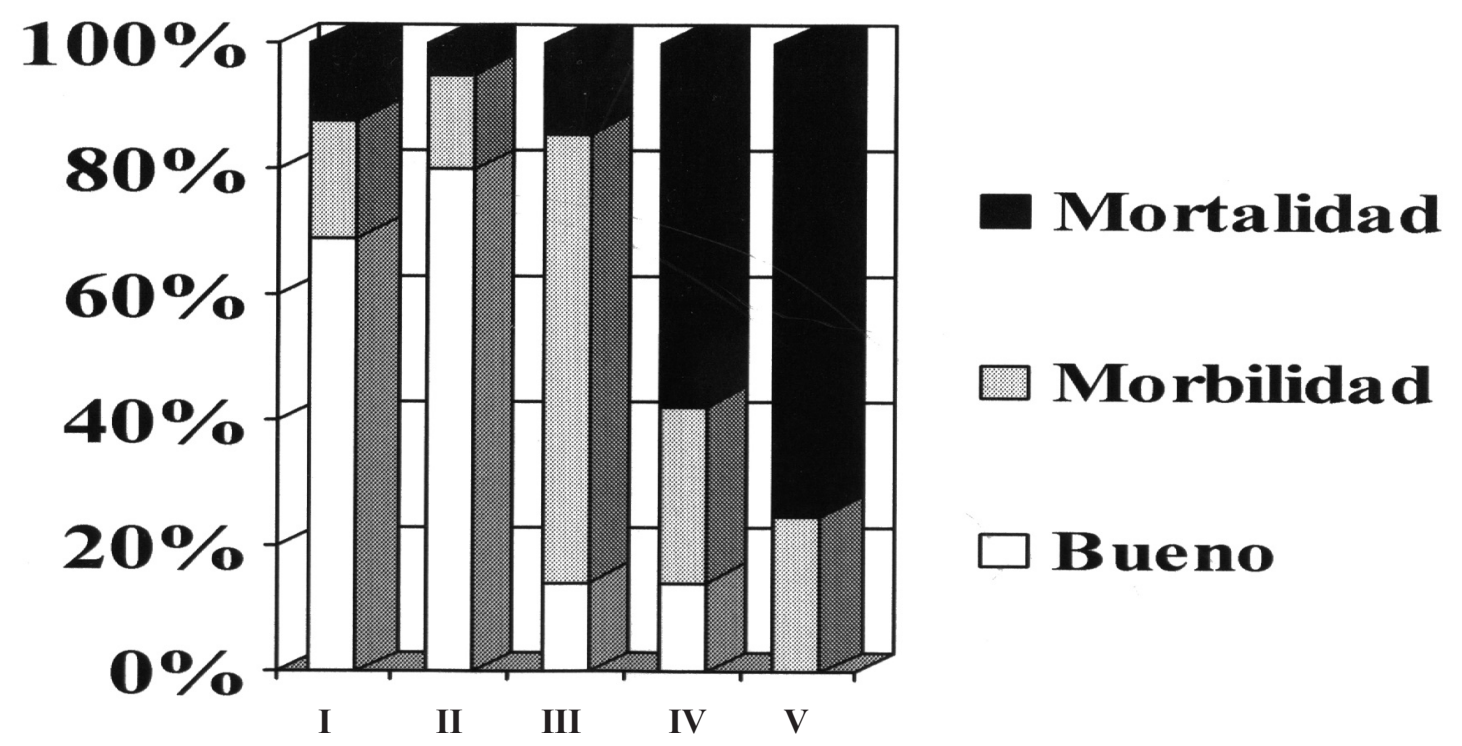

Figura 3. Relación entre grado de Hunt-Hess y pronóstico.

de ellos se debió a vasoespasmo y a resangrado en 4 . La frecuencia de vasoespasmo clínico fue del $20 \%$ y la de resangrado del $16 \%$.

Las características de los pacientes se muestran en la tabla 2.

Tratamiento. Se pudo llevar a cabo embolización en 28 casos (54\%). Hubo necesidad de intervención quirúrgica, debido a las características del aneurisma, en 21 (38\%), y ésta fue precoz en 14 (70\%).

Cinco pacientes no fueron tratados por presentar HSA devastadora inicial, de los que fallecieron 4.

Seis pacientes $(16,8 \%)$ embolizados y $3(14 \%)$ operados precozmente tuvieron secuelas neurológicas ( $\mathrm{p}>$ $0.05)$. Fallecieron $3(14,2 \%)$ enfermos con tratamiento endovascular y $4(19 \%)$ quirúrgicos precoces ( $\mathrm{p}>0.05)$.

La estancia media en UCI de los pacientes con tratamiento quirúrgico fue de 8,8 días; rango 1-40 días y la de los pacientes embolizados de 6,1 días; rango de 1-30 días. $(\mathrm{p}<0.05)$ Tabla 3.

\section{Limitaciones del estudio}

Es un estudio retrospectivo con pocos pacientes. La terapia endovascular está evolucionando rápidamente en el tiempo, por lo que los pacientes tratados más recientemente pueden tener mejor pronóstico que los incluidos en este estudio.

\section{Discusión}

Este artículo detalla nuestra experiencia en el tratamiento de 54 pacientes con aneurismas rotos en tres años.

La HSA supone el $5-10 \%$ de todos los ictus y a pesar de la evolución de las técnicas diagnósticas, quirúrgicas, endovasculares y anestésicas siguen caracterizándose por su alta morbi-mortalidad, con $45 \%$ de los pacientes que

Tabla 1

Resultado en relación con la localización del aneurisma

\begin{tabular}{llll}
\hline & $\begin{array}{l}\text { Buen resultado } \\
\mathrm{N}^{\circ} \text { pacientes } / \%\end{array}$ & $\begin{array}{l}\text { Morbi-mortalidad } \\
\mathrm{N}^{\circ} \text { pacientes } / \%\end{array}$ & \\
\hline Circulación posterior & $8(53.3 \%)$ & $7(46.6 \%)$ & 15 \\
Circulación anterior & $21(53.84 \%)$ & $18(46.16 \%)$ & 39 \\
Total & 29 & 25 & 54 \\
\hline
\end{tabular}

$\mathbf{P}>\mathbf{0 . 0 5}$ 
Tabla 2

Características y tratamiento aplicado en los pacientes

\begin{tabular}{llll}
\hline & Embolización & Cirugía precoz & p valor \\
\hline Edad media & $55.4+/-35.2$ & $50.2+/-13.7$ & NS \\
Mujer ( ${ }^{\circ}$ pacientes $)$ & $17(60.7 \%)$ & $10(47.6 \%)$ & NS \\
APACHE II $>12\left(\mathrm{n}^{\circ}\right.$ pacientes $)$ & 13 & 5 & $\mathrm{NS}$ \\
Hunt-Hess I-III $\left(\mathrm{n}^{\circ}\right.$ pacientes $)$ & 23 & 17 & $\mathrm{NS}$ \\
Hunt-Hess IV-V $\left(\mathrm{n}^{\circ}\right.$ pacientes $)$ & 5 & 4 & $\mathrm{NS}$ \\
\hline
\end{tabular}

* NS: no significativo estadísticamente $(\mathrm{p}>0.05)$

* Wilcoxon y $\chi^{2}$

Tabla 3

Resultados en pacientes embolizados y sometidos a cirugía convencional

\begin{tabular}{llll}
\hline & Embolizados & Quirúrgicos precoces & $\mathrm{p}$ valor \\
\hline Estancia en UCI & 6.1 días (1-30) & 8.8 días $(1-40)$ & $\mathrm{p}<0.05$ \\
Mortalidad & $\mathrm{n}=3(14.2 \%)$ & $\mathrm{n}=4(19 \%)$ & $\mathrm{p}>0.05$ \\
Morbilidad & $\mathrm{n}=6(16.8 \%)$ & $\mathrm{n}=3(14.2 \%)$ & $\mathrm{p}>0.05$ \\
\hline
\end{tabular}

Tabla 4

Resultados del manejo global

$$
\text { Buen resultado }\left(\mathrm{n}^{\circ} \text { pacientes }\right) \quad \text { Morbi-mortalidad }\left(\mathrm{n}^{\circ} \text { pacientes }\right)
$$

Tratamiento precoz

Tratamiento tardío

$* \mathrm{p}<0.05$

* El tratamiento precoz parece ser superior cuando se analizan los resultados del manejo global. fallecen en el primer mes y un 50\% que queda con secue$\operatorname{las}^{5,9}$.

Nosotros encontramos una morbi-mortalidad del 46\%, la cual es relativamente baja comparada con publicaciones previas $^{12}$ y una mortalidad del $20 \%$, similar a la encontrada por Qureshi ${ }^{17}$ e Ikawa ${ }^{10}$.

La comparación con series quirúrgicas es complicada porque las poblaciones estudiadas son diferentes, así como la metodología de evaluación del seguimiento de los pacientes.

Una de las posibles causas de esta mejoría puede ser el incremento de pacientes que son atendidos en el Centro de Referencia en las primeras 24 horas; en nuestro caso fue del $86 \%$. En el estudio de Seiler ${ }^{22}$ solo un $67 \%$ llegaba al hospital en las primeras 24 horas del sangrado.
Un incremento en el nivel de cuidados intensivos también contribuye a la mejoría de los resultados, junto con la oclusión precoz del aneurisma. El tratamiento precoz parece ser superior al tardío cuando se analizan los resultados del manejo global. Tabla 4.

Encontramos mejores resultados en los pacientes tratados en los primeros 5 días de ocurrida la HSA, hallazgos similares a los de otros artículos (Kassell) ${ }^{11}$, donde concluían que la cirugía precoz $(<10$ días después del sangrado) no era técnicamente más dificil que la tardía y que esperar más de 10 días supone un aumento de la incidencia de complicaciones (resangrado).

En algunas series previas se ha comparado el pronóstico de los pacientes bajo los dos tipos de tratamiento. En un estudio randomizado con pocos pacientes de Vanninen ${ }^{24}$ 
no se observan diferencias en cuanto al pronóstico entre ambos procedimientos. En el estudio de Rabinstein ${ }^{19}$ se hizo una revisión de 415 pacientes con HSA aneurismática que habían sido tratados con oclusión endovascular o craneotomía, aunque encuentran que los pacientes con mejores grados clínicos a la llegada al Hospital (WFNS I-II ) sufrieron menos vasoespasmo cuando se trataron con oclusión con coils comparado con craneotomía y aplicación de clips; no se encontraron diferencias significativas en el pronóstico tras seguimiento a largo plazo entre los 2 tipos de tratamiento.

Resultados similares son hallados por $\mathrm{Hoh}^{7}$, en estudio retrospectivo en un único centro, no randomizado; observa que la utilización de clips y/o craneotomía tiene mejor pronóstico y menos mortalidad al alta que la embolización en pacientes con buen grado de Hunt-Hess pero no encuentra diferencias en el riesgo de vasoespasmo entre pacientes tratados con craneotomía y clip, comparado con el grupo tratado con embolización, en pacientes con buen o mal grado de Hunt y Hess.

En el mismo sentido se manifiesta Goddard ${ }^{4}$ en las conclusiones de su estudio: "El método de tratamiento no tiene influencia en la incidencia o duración de vasoespasmo y no hay diferencia significativa en el pronóstico al alta y seguimiento entre los 2 tipos de tratamiento".

El estudio ISAT ${ }^{14}$ demostró que sí había diferencias estadísticamente significativas entre los grupos : $22,7 \%$ de pacientes tratados con coils fueron dependientes o muertos comparado con $30,6 \%$ de los tratados quirúrgicamente. Se han hecho varias objeciones a este estudio, entre ellas: de los no elegidos, la mayoría se trataron con intervención quirúrgica en vez de embolización. Los equipos neurovasculares de los Centros participantes consideraron que la cirugía era la mejor opción para la mayoría de los pacientes no randomizados ${ }^{2,6}$. Cinco Centros de Reino Unido incorporaron al estudio aproximadamente la mitad de los pacientes. Esto ha llevado a poner en duda la aplicabilidad de los resultados en Centros de otras partes del mundo ${ }^{18,21}$.

Las condiciones en las que se hizo el estudio ISAT (pacientes con buen grado de Hunt-Hess, aneurismas pequeños, de circulación anterior y con posibilidad de ser tratados tanto con cirugía como con embolización), no son las mismas con las que trabajamos habitualmente en nuestro Centro. En el Hospital de León se usa la terapia endovascular como primera opción y se lleva a cabo cirugía si el tratamiento mediante coils no se considera posible. En nuestro caso el resultado de los dos tipos de tratamiento es el mismo.

Es necesario considerar que el tratamiento endovascular requiere arteriografía y a menudo otro tratamiento. La frecuencia, eficacia y riesgos asociados con este nuevo tratamientos y, lo más importante, la frecuencia de resangrado está aún por determinar.

\section{Conclusión}

La embolización se pudo llevar a cabo con éxito en más de la mitad de los casos, a pesar de que el estudio está hecho al inicio de la técnica endovascular en nuestro hospital.

No encontramos diferencias estadísticamente significativas, en cuanto a pronóstico, entre la embolización y el tratamiento quirúrgico. Pero sí que hay relación entre el nivel de conciencia al ingreso y el resultado.

Hay que tener en cuenta que los resultados de la embolización no son estáticos; están mejorando constantemente; no solo nuestros radiólogos intervencionistas están ganando más experiencia, sino que también la microtecnología de las técnicas endovasculares mejora y evoluciona con el tiempo.

Lo que sí vemos claramente es una reducción de la estancia en UCI de los pacientes embolizados frente a los quirúrgicos, como en otros estudios anteriores ${ }^{13}$.

La cirugía llevada a cabo por neurocirujanos con experiencia es un método seguro de tratamiento de los aneurismas intracraneales ${ }^{15}$. El pronóstico parece depender principalmente del grado inicial de Hunt-Hess del paciente.

Es imprescindible la colaboración entre neurocirujanos y radiólogos en el manejo de los pacientes con HSA debida a rotura de aneurismas, planificando e individualizando el tratamiento.

Algunos estudios han probado que la historia natural de los aneurismas rotos, tratados de forma conservadora, es relativamente benigna. Alvord et $\mathrm{al}^{1}$ mostraron que los pacientes que sobreviven entre 1 y 3 días en grado I de Hunt-Hess, después de la rotura de un aneurisma, tenían un $80 \%$ de probabilidades de estar vivos a los 2 años, mientras que si sobrevivían entre 7 y 21 días la probabilidad es de $95 \%$. Sharr y Kelvin ${ }^{23}$ encontraron que la gran mayoría de los aneurismas vertebrobasilares tratados de forma conservadora permanecen bien muchos años más tarde. El estudio de Nishioka ${ }^{16}$ en los años 60 encuentra que de 34 pacientes que rechazaron operación por rotura de aneurismas, el $82 \%$ permanecía vivo y bien en el seguimiento a los 6 años.

Un manejo integrado aportando cuidados críticos antes, durante y después de la oclusión del aneurisma debe ser el estándar. La observación neurológica estrecha y técnicas especializadas de monitorización son necesarias para conseguir tratamiento intensivo adecuado en el periodo agudo después de una HSA.

El debate y la controversia sobre qué tratamiento es el más adecuado, si endovascular o quirúrgico, permanecerá por ahora, sabiendo que algunos aneurismas requieren los dos tipos de tratamiento. 


\section{Bibliografía}

1. Alvord, E.C., Loeser, J.D., Bailey, W.L., et al.: Subarachnoid haemorrhage due to ruptured aneurysms. A simple method of estimating prognosis. Arch Neurol 1972; 27: 273291.

2. Britz, G.W., Newell, D.W., West, G.A., Lam, A.: The ISAT trial. Lancet 2003; 361: 431-432.

3. Fisher, C.M., Kistler, J.P., Davis, J.M.: Relation of cerebral vasospasm to subarachnoid hemorrahg visualized by computer tomographic scanning. Neurosurgery 1980; 6: 1-9.

4. Goddard, A.J.P., Raju, P.P.J., Gholkar, A.: Does the method of treatment of acutely ruptured intracranial aneurysms influence the incidence and duration of cerebral vasospasm and clinical outcome? J Neurol Neurosurg Psychiatry 2004; 75: 868-872.

5. Graves, E.J.: Detailed diagnoses and procedures, National Hospital Discharge Survey 1990. Vital Health Stat.13 1992; 113: 1-225.

6. Harbaugh, R.E., Heros, R.C., Hadley, M.N.: More on ISAT. Lancet 2003; 361: 783-784.

7. Hoh, B.L., Topcuoglu, M.A., Singhal, A.B., et al.: Effect of clipping, craniotomy, or intravascular coiling on cerebral vasospasm and patient autcome alter aneurysmal subarachnoid hemorrhage. Neurosurgery 2004; 55: 779-789.

8. Hunt, W.E. and Hess, R.M.: Surgical risk as related to time of intervention in the repair of intracranial aneurysrns. J. Neurosurgery $1968 ; 28: 14-19$.

9. Ingall, Tj., Wiebers, D.O.: Natural history of subarachnoid hemorrhage. Wishna,t J..P ed. Stroke: populations, cohorts, and clinical trials. Boston, Mass: Butterworth Heinemann Ltd 1993; 174-186.

10. Ikawa, F., Ohbasyashi, N., Imada, Y., et al.: Analysis of subarachnoid hemorrhage according to the japanese standard stroke registry study -incidence, outcome, and comparison with the international subarachnoid aneurysm trial. Neurol Med Chir (Tokyo) 2004; 44: 275-276.

11. Kasell, N.F., Tourer, J.C., Haley, C. et al.: The International Cooperative Study on the Timing of Aneurysm Surgery. J. Neurosurgery 1990; 73: 37-47.

12. Ljunggren, B., Fodstad, H., von Essen, C., et al.: Aneurismal subaracnoid hemorrhage: overall outcome and incidence of early recurrent hemorrhage despite a policy of acute stage operation. Br J Neurosurgery 1988; 2: 49-53.

13. Martínez-Mañas, R., Ibañez, G., Macho, J., Gastón, F., Ferrer, E.: Estudio de 234 pacientes con hemorragia subaracnoidea de origen aneurismático y criptogenético. Neurocirugía 2002; 13: 281-295.

14. Molyneux, A., Kerr, R., Stratton, I., Sandercock, P., et al.: International Subarachnoid Aneurysm Trial (ISAT) Collaborative Group: International Subarachnoid Aneurysm Trial (ISAT) of neurosurgical clipping versus endovascular coiling in 2143 patients with ruptured intracranial aneurysms: a randomised trial. Lancet 2002; 360: 1267-1274.

15. Morales, F., Maillo, A., Hernández J., et al.: Evaluación del tratamiento microquirúrgico de 121 aneurismas intracraneales. Neurocirugía 2003; 14: 5-15.

16. Nishioka, H.: Evaluation of the conservative management of ruptured intracraneal aneurysms. J Neurosurgery 1966; 10: 129-143.

17. Qureshi, A.I. Suárez, J.I., Bhardwaj, A., et al.: Early predictors of outcome in patients receiving hypervolemic and hypertensive therapy for symptomatic vasospasm after subarachnoid hemorrhage. Crit Care Med 2000; 28: 824829.

18. Raabe, A., Schmiedek, P., Seifert, V., Stolk, D.: Position Statement on the International Subarachnoid hemorrhage Trial (ISAT). Zentralbl Neurochir 2003; 64: 99-103.

19. Rabinstein, A.A., Pichelmann, M.A., Friedman, J.A., et al.: Symptomatic vasospasm and outcomes following aneurismal sub arachnoid hemorrhage: a comparison between surgical repair and endovascular coil occlusion. J. Neurosurg 2003; 98: 319-325.

20. Roda, J.M., Conesa, G., Díez Lobato, R., et al.: Hemorragia subaracnoidea aneurismática. Introducción a algunos aspectos más importantes de esta enfermedad. Neurocirugía 2000; 11: 156-168.

21. Sade, B., Mohr, G.: Critical appraisal of the International Subarachnoid Aneurysm Trial (ISAT). Neurol India 2004; 52: $32-35$.

22. Seiler, R.W., Reulen, H.J., Huber, P., Grolimund, P., Ebeling, U., Steiger, H.: Outcome in aneurismal subarachnoid in a hospital population: a prospective study including early operation, intravenous nimodipine and transcranial Doppler ultrasound. Neurosurgery 1998; 23: 598-604.

23. Sharr, M.M., Kelvin, F.M.: Vertebro-basilar aneurysms: experience with 27 cases. Euro. Neurol. 1973; 10: 129143.

24. Vanninen, R., Koivisto, T., Saari, T., Hernesniemi, J., Vapalahti, M.: Ruptured intracranial aneurysms: acute endovascular treatment with electrlytically detachable coils - a prospective randomized study. Radiology 1999; 211: 325-336.

25. Vespa, P.M-, Gobin, Y.P.: Endovascular treatment and neurointensive care of ruptured aneurysms. Critical Care Clinics 1999; 15: 667-683.

González-Pérez, Mà.I.: Resultado del tratamiento de la hemorragia subaracnoidea debida a rotura de aneurismas cerebrales. Neurocirugía 2006; 17: 433-439.

Correspondencia postal: María Isabel González Pérez. Servicio de Medicina Intensiva del Complejo Hospitalario de León. Altos de Nava s/n. 24071 León. 